\title{
Effects of the Neutron Spin-Orbit Density on Nuclear Charge Density in Relativistic Models
}

\author{
Haruki Kurasawa \\ Department of Physics, Faculty of Science, Chiba University, Chiba 263-8522, Japan \\ Toshio Suzuki \\ Department of Applied Physics, Fukui University, Fukui 910-8507, Japan \\ RIKEN, 2-1 Hirosawa, Wako-shi, Saitama 351-0198, Japan
}

\begin{abstract}
The neutron spin-orbit density contributes to the nuclear charge density as a relativistic effect. The contribution is enhanced by the effective mass stemming from the Lorentzscalar potential in relativistic models. This enhancement explains well the difference between the cross sections of elastic electron scattering off ${ }^{40} \mathrm{Ca}$ and ${ }^{48} \mathrm{Ca}$ which was not reproduced in non-relativistic models. The spin-orbit density will be examined in more detail in electron scattering off unstable nuclei which would be available in the future.

PACS numbers: 21.10.Ft, 25.30.Bf
\end{abstract}

At present there are two kinds of phenomenological models which explain well nuclear structure and reactions. The one is a conventional non-relativistic model which assumes phenomenological interactions between nucleons, like Skyrme forces [1]. The other is a relativistic model which takes into account explicitly mesonexchanges between nucleons, as the $\sigma-\omega$ model [2]. The relationship between these models, however, is not clear. In the relativistic model the effective mass coming from the Lorentz scalar potential, which is absent in nonrelativistic models, plays a crucial role in understanding nuclear properties. For example, the effective mass enhances the spin-orbit force which is responsible for the spin-orbit splitting of the single-particle levels and polarization phenomena of hadron-nucleus scattering. The nuclear saturation density and binding energy are also dominated by the effective mass. In non-relativistic models those quantities are explained by taking into account other kinds of many-body correlations. It is one of the important questions in nuclear physics which model is realistic. The best way to answer this question is to find fundamental physical quantities which can be explained in the one model, but not in the other model. So far, however, such quantities are not found, as far as the authors know.

The purpose of this paper is to show that the neutron spin-orbit density is very sensitive to the effective mass of the relativistic model. The effective mass yields an additional spin-orbit density as a relativistic correction, which contributes to the nuclear charge density, and the effects are seizable in the form factors of elastic electron scattering. This correction explains well the existing data which were not reproduced in previous non-relativistic models [3]. We will also show that the effective mass effects will be able to be explored in more detail in unstable nuclei.

The neutron spin-orbit charge density is due to the Pauli current. It was first discussed by Bertozzi et al. [3] in a non-relativistic framework. The nuclear wave function was obtained in a two-component model and the relativistic correction was derived by expanding the free nucleon current in terms of $1 / M, M$ being the mass of the free nucleon. They calculated the cross sections for elastic electron scattering off ${ }^{40} \mathrm{Ca}$ and ${ }^{48} \mathrm{Ca}$, and found that the relativistic correction was not negligible, but not enough to explain the difference between the two cross

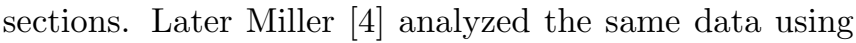
a relativistic model, but again could not reproduce the experimental data. The Pauli current made rather worse the agreement between his results and experiment.

Our model discussed below is in principle the same as Miller's one, but we will calculate the spin-orbit density, using a different relativistic model developed later by Horowitz and Serot [5]. Moreover we will make clear the relationship between the relativistic model and nonrelativistic models. The effective mass effects, which are peculiar to the relativistic model, on the spin-orbit current will be clarified.

We calculate the cross section for elastic electron scattering using phase shift analyses [6]. For this purpose we have to obtain the nuclear charge density, which is given by

$$
\rho_{c}(r)=\int \frac{d^{3} q}{(2 \pi)^{3}} \exp (-i \mathbf{q} \cdot \mathbf{r})\langle 0|\hat{\rho}(\mathbf{q})| 0\rangle,
$$

where $\mathbf{q}$ denotes the momentum transfer from the electron to the nucleus. In the relativistic theory, the groundstate expectation value of the time-component of the nuclear current is given by

$$
\begin{aligned}
\langle 0|\hat{\rho}(\mathbf{q})| 0\rangle= & \langle 0| \sum_{k} \exp \left(i \mathbf{q} \cdot \mathbf{r}_{k}\right) \\
& \times\left(F_{1 k}\left(\mathbf{q}^{2}\right)+\frac{\mu_{k}}{2 M} F_{2 k}\left(\mathbf{q}^{2}\right) \mathbf{q} \cdot \gamma_{k}\right)|0\rangle,
\end{aligned}
$$

where $F_{1 k}\left(\mathbf{q}^{2}\right)$ and $F_{2 k}\left(\mathbf{q}^{2}\right)$ stand for the Dirac and Pauli form factors of the nucleon, respectively, and $\mu_{k}$ the anomalous magnetic moment. The above equation is rewritten by using the Sachs form factor, $G_{E}\left(\mathbf{q}^{2}\right)$, as 


$$
\begin{aligned}
\langle 0|\hat{\rho}(\mathbf{q})| 0\rangle= & \int d^{3} x \exp (i \mathbf{q} \cdot \mathbf{x}) \\
& \times \sum_{\tau}\left(G_{E \tau}\left(\mathbf{q}^{2}\right) \rho_{\tau}(x)+F_{2 \tau}\left(\mathbf{q}^{2}\right) W_{\tau}(x)\right) \\
= & \int d^{3} x d^{3} y \exp (i \mathbf{q} \cdot(\mathbf{x}+\mathbf{y})) \\
& \times \sum_{\tau}\left(G_{E \tau}(y) \rho_{\tau}(x)+F_{2 \tau}(y) W_{\tau}(x)\right),
\end{aligned}
$$

where the sum of $\tau$ is performed with respect to the proton and the neutron, $\tau=p, n$. The functions, $G_{E \tau}(y)$ and $F_{2 \tau}(y)$, are obtained by the inverse Fourier transformation of the Sachs and Pauli form factors, respectively. The nucleon density, $\rho_{\tau}(x)$, and the spin-orbit density, $W_{\tau}(x)$, are given by

$$
\begin{aligned}
\rho_{\tau}(r)= & \left\langle 0\left|\sum_{k} \delta\left(\mathbf{r}-\mathbf{r}_{k}\right)\right| 0\right\rangle, \\
W_{\tau}(r)= & \frac{\mu_{\tau}}{2 M}\left(-\frac{1}{2 M} \nabla^{2} \rho_{\tau}(r)\right. \\
& \left.+i \boldsymbol{\nabla} \cdot\left\langle 0\left|\sum_{k} \delta\left(\mathbf{r}-\mathbf{r}_{k}\right) \boldsymbol{\gamma}_{k}\right| 0\right\rangle\right),
\end{aligned}
$$

where the sum over $k$ is performed up to $Z$ for $\tau=p$ and up to $N$ for $\tau=n$. By inserting Eq. (3) into Eq. (11), the nuclear charge density is given by

$$
\rho_{c}(r)=\sum_{\tau}\left(\rho_{c \tau}(r)+W_{c \tau}(r)\right),
$$

where the nucleon charge density, $\rho_{c \tau}(r)$, and the spinorbit charge density, $W_{c \tau}(r)$, are written as

$$
\begin{aligned}
\rho_{c \tau}(r)= & \frac{1}{r} \int_{0}^{\infty} d x x \rho_{\tau}(x) \\
& \times\left(g_{\tau}(|r-x|)-g_{\tau}(r+x)\right), \\
W_{c \tau}(r)= & \frac{1}{r} \int_{0}^{\infty} d x x W_{\tau}(x) \\
& \times\left(f_{2 \tau}(|r-x|)-f_{2 \tau}(r+x)\right)
\end{aligned}
$$

with

$$
\begin{aligned}
& g_{\tau}(x)=\frac{1}{2 \pi} \int_{-\infty}^{\infty} d q e^{i q x} G_{E \tau}\left(\mathbf{q}^{2}\right), \\
& f_{2 \tau}(x)=\frac{1}{2 \pi} \int_{-\infty}^{\infty} d q e^{i q x} F_{2 \tau}\left(\mathbf{q}^{2}\right) .
\end{aligned}
$$

In order to calculate the nucleon and the spin-orbit density, we take the Horowitz-Serot model [5], where the ground state wave function is described with the mean field approximation. The single-particle wave function is written as

$$
\psi_{\alpha m}=\left(\begin{array}{c}
i \frac{G_{\alpha}(r)}{r} \mathcal{Y}_{\ell j m} \\
\frac{F_{\alpha}(r)}{r} \frac{\boldsymbol{\sigma} \cdot \mathbf{r}}{r} \mathcal{Y}_{\ell j m}
\end{array}\right),
$$

The large and small components in the present model satisfy the Dirac equation:

$$
\begin{aligned}
\frac{d G_{\alpha}}{d r} & =-\frac{\kappa_{\alpha}}{r} G_{\alpha}+\left(\varepsilon_{\alpha}-U_{\tau}(r)+M^{*}(r)\right) F_{\alpha}, \\
\frac{d F_{\alpha}}{d r} & =\frac{\kappa_{\alpha}}{r} F_{\alpha}-\left(\varepsilon_{\alpha}-U_{\tau}(r)-M^{*}(r)\right) G_{\alpha},
\end{aligned}
$$

where $\kappa_{\alpha}=(-1)^{j-\ell+1 / 2}(j+1 / 2)$ denotes the eigenvalue of $-(1+\boldsymbol{\sigma} \cdot \boldsymbol{\ell})$, and $M^{*}(r)$ the nucleon effective mass given by

$$
M^{*}(r)=M-U_{s}(r) .
$$

The Lorentz scalar potential, $U_{s}(r)$, comes from the $\sigma$ meson exchanges between nucleons, while the Lorentz vector potential, $U_{\tau}(r)$, is due to the $\omega$ - and $\rho$-mesons and photons in the present model. Then the nucleon density in Eq. (四) is given by

$$
\rho_{\tau}(r)=\sum_{\alpha} \frac{2 j_{\alpha}+1}{4 \pi r^{2}}\left(G_{\alpha}^{2}+F_{\alpha}^{2}\right) .
$$

On the other hand, the spin-orbit density, $W_{\tau}(r)$, in Eq. (5) is described as

$$
\begin{aligned}
W_{\tau}(r)= & \frac{\mu_{\tau}}{M} \sum_{\alpha} \frac{2 j_{\alpha}+1}{4 \pi r^{2}} \frac{d}{d r}\left(\frac{M-M^{*}(r)}{M} G_{\alpha} F_{\alpha}\right. \\
& \left.+\frac{\kappa_{\alpha}+1}{2 M r} G_{\alpha}^{2}-\frac{\kappa_{\alpha}-1}{2 M r} F_{\alpha}^{2}\right) .
\end{aligned}
$$

The relationship between the relativistic model and non-relativistic models is very clear. In non-relativistic models, usually the neutron charge and spin-orbit charge densities are neglected in estimating the electron scattering cross section. Bertozzi et al. [3] took into account the neutron charge density and a part of the spin-orbit charge density in the non-relativistic framework. Their spin-orbit density is obtained from Eq. (11) by setting $M^{*}(r)=M$ and neglecting $F_{\alpha}^{2}$-term,

$$
\begin{aligned}
W_{\tau}(r) & \approx \frac{\mu_{\tau}}{2 M^{2} r^{2}} \frac{d}{d r} r \sum_{\alpha} \frac{2 j_{\alpha}+1}{4 \pi r^{2}}\left(\kappa_{\alpha}+1\right) G_{\alpha}^{2} \\
& \approx-\frac{1}{r^{2}} \frac{d}{d r} r\left\langle 0\left|\frac{\mu_{\tau}}{2 M^{2}} \sum_{k} \delta\left(\mathbf{r}-\mathbf{r}_{k}\right) \boldsymbol{\sigma}_{k} \cdot \boldsymbol{\ell}_{k}\right| 0\right\rangle .
\end{aligned}
$$

We will show that the spin-orbit density due to the effective mass in Eq. (11) is very important in the relativistic model for reproducing the experimental data.

The nucleon form factors used in the present calculations are the dipole type according to Ref. 3] with more recent experimental data for the neutron [7]. The Sachs form factor for the proton is given by

$$
G_{E p}=\frac{1}{\left(1+r_{p}^{2} \mathbf{q}^{2} / 12\right)^{2}}, \quad r_{p}=\left\langle r^{2}\right\rangle^{1 / 2}=0.81 \mathrm{fm},
$$


while the one for the neutron by

$$
\begin{aligned}
G_{E n} & =\frac{1}{\left(1+r_{+}^{2} \mathbf{q}^{2} / 12\right)^{2}}-\frac{1}{\left(1+r_{-}^{2} \mathbf{q}^{2} / 12\right)^{2}}, \\
r_{ \pm}^{2} & =(0.9)^{2} \mp 0.06 \mathrm{fm}^{2} .
\end{aligned}
$$

The Pauli form factors for the proton and the neutron are

$$
F_{2 p}=\frac{G_{E p}}{1+\mathbf{q}^{2} / 4 M^{2}}, \quad F_{2 n}=\frac{G_{E p}-G_{E n} / \mu_{n}}{1+\mathbf{q}^{2} / 4 M^{2}} .
$$

The values of the anomalous magnetic moment are given by $\mu_{p}=1.793$ and $\mu_{n}=-1.913$.

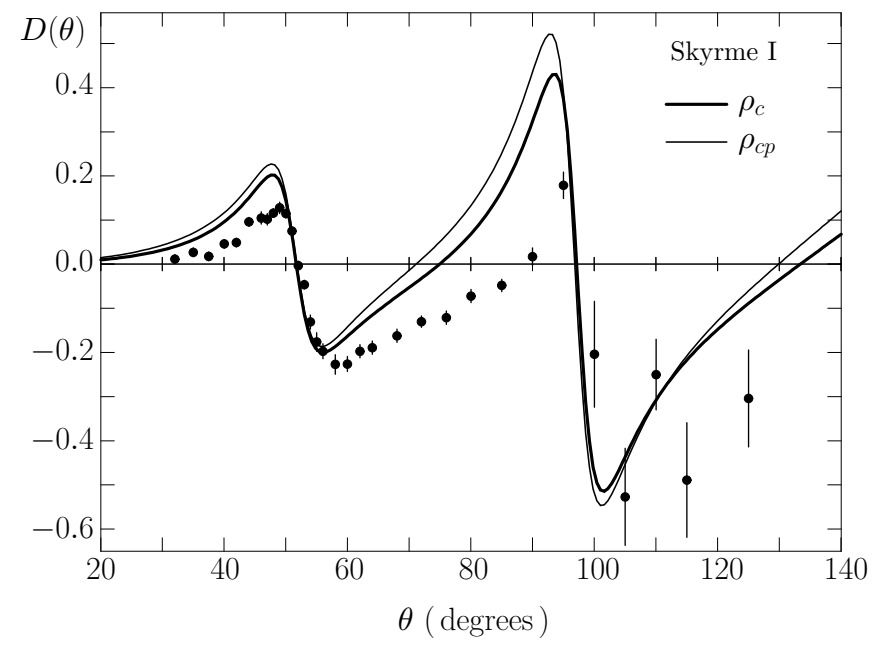

FIG. 1. The difference $D(\theta)$ for ${ }^{40} \mathrm{Ca}$ and ${ }^{48} \mathrm{Ca}$ given by Eq. (14) as a function of scattering angle $\theta$ for elastic electron scattering at $250 \mathrm{MeV}$. Numerical values are calculated by the non-relativistic Hartree-Fock approximation with the Skyrme force I. The thin solid curve shows the results with the only proton charge density, while the solid curve with the full charge density. Experimental data points are taken from Ref. [8].

Now, we calculate the differential cross sections $\sigma(\theta)$ for elastic electron scattering off ${ }^{40} \mathrm{Ca}$ and ${ }^{48} \mathrm{Ca}$ and compare their difference $D(\theta)$ given by

$$
D(\theta)=\frac{\sigma_{40}(\theta)-\sigma_{48}(\theta)}{\sigma_{40}(\theta)+\sigma_{48}(\theta)}
$$

with experiment. First we show in Fig. 1 results of the non-relativistic models, since Bertozzi et al. [3] did not compare explicitly their results including both the nucleon charge density and the spin-orbit charge density with experiment. In Fig. 1, the thin solid curve shows the result taking into account the only proton charge density, which is calculated with the Skyrme force I in the Hartree-Fock approximation [1]. This is almost the same as the result of Bertozzi et al. [3]. When we include also the neutron charge density and the proton and neutron spin-orbit charge density in Eq. (13), we obtain the solid curve in Fig. 1. It is seen that the the discrepancy between the theory and the experiment is a little reduced, in particular, at the electron scattering angle around $\theta=60^{\circ}$ to $90^{\circ}$. The improvement is mainly due to the spin-orbit charge density from the neutrons in the $1 f_{7 / 2}$ shell, but is not enough to explain the experimental data 8 .

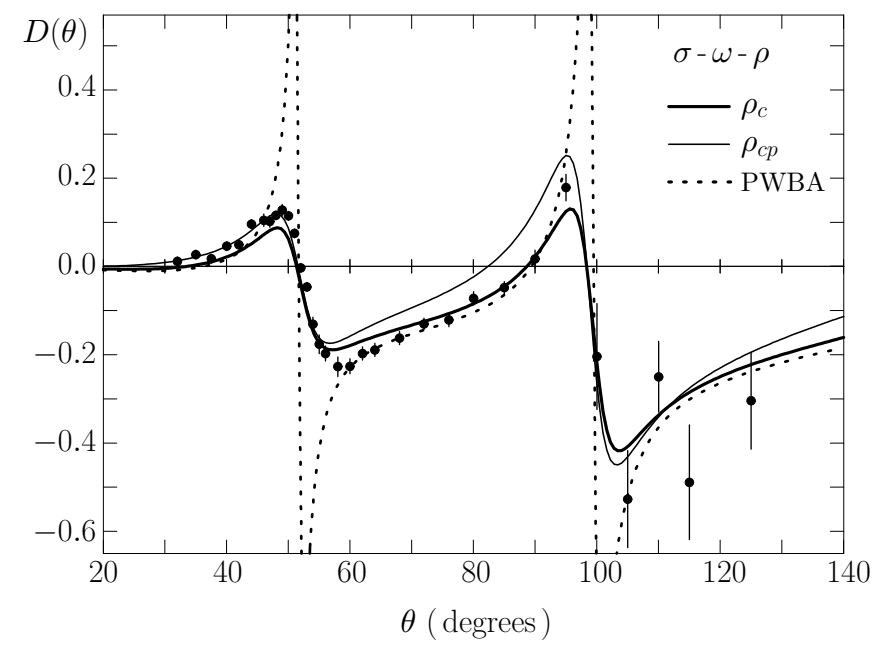

FIG. 2. Same as Fig. 1, for the numerical results of the relativistic model. The dotted curve is calculated in PWBA with the effective momentum transfer.

Fig. 2 shows the results of the Horowitz-Serot model [5]. The thin solid curve is obtained by taking into account the only proton charge density, while the solid curve by the full density, Eq. (5). In the relativistic model, the proton charge density itself improves results of the non-relativistic model, and the experimental data are almost reproduced by taking into account the neutron spin-orbit charge density enhanced by the effective mass. In this figure, we show, as a reference, by the dotted curve the results of the PWBA calculations using the full density and the effective momentum transfer to simulate DWBA. We note that both in non-relativistic and relativistic models, the center of mass correction to the cross section are negligible in Ca isotopes [9].

As seen in Eq. (11), the effects of the spin-orbit charge density appears, when the sub-shell is occupied by the neutrons; In closed shell nuclei, the effects disappear. Moreover, if protons also occupy the subshell as in ${ }^{208} \mathrm{~Pb}$, the proton and neutron spin-orbit densities almost cancel each other as in non-relativistic model [3], since the anomalous magnetic moment of the proton has the opposite sign to that of the neutron. Another interesting result of the spin-orbit density is found in neutron rich nuclei. In Fig. 3 shown the results with respect to ${ }^{40} \mathrm{Ca}$ and ${ }^{52} \mathrm{Ca}$ in the same designation as in Fig. 2. We see that effects from the spin-orbit charge density of the $1 f_{7 / 2}$ neutrons are almost cancelled by those from the $2 p_{3 / 2}$ neutrons. The similar results are obtained in $\mathrm{Zr}$ isotopes 
[9]. The effect of the neutron spin-orbit charge density is enhanced in the cross section of ${ }^{90} \mathrm{Zr}$, compared with the one of ${ }^{80} \mathrm{Zr}$, but disappears in ${ }^{96} \mathrm{Zr}$. It is interesting to observe experimentally these predictions of the relativistic model in electron scattering off unstable nuclei which is planned in RIKEN [10].

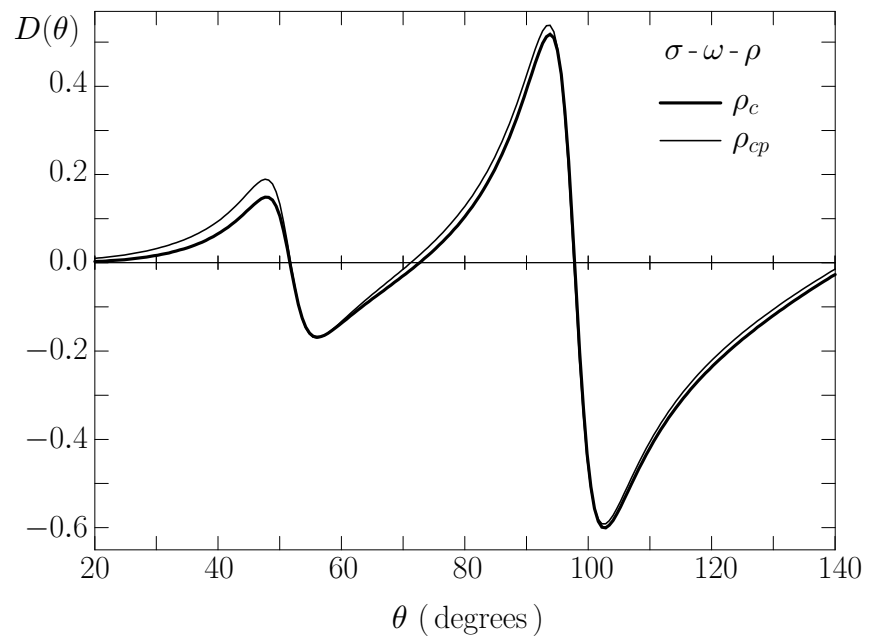

FIG. 3. Same as Fig. 2, for the difference as to ${ }^{40} \mathrm{Ca}$ and ${ }^{52} \mathrm{Ca}$ calculated by the relativistic model.

In conclusion, the effective mass due to the Lorentz scalar potential is a necessary ingredient of the relativistic model. It enhances the neutron spin-orbit charge density in a peculiar way to this model. The enhanced density explains well the difference between the cross sections of elastic electron scattering off ${ }^{40} \mathrm{Ca}$ and ${ }^{48} \mathrm{Ca}$, which was not reproduced in previous non-relativistic models. Electron scattering off unstable nuclei is desirable in order to explore in more detail the effective mass effects. More detailed discussions on the results of non-relativistic and relativistic models will be published elsewhere [9].

\section{ACKNOWLEDGMENTS}

The authors would like to thank Dr. T. Suda for useful discussions.
[6] D. R. Yennie, D. G. Ravenhall and R. N. Wilson, Phys. Rev. 95 (1954) 500.

[7] T. Eden et al., Phys. Rev. C50 (1994) 1749; M. Meyerhoff et al., Phys. Lett. 327B (1994) 201; S. Platchkov et al., Nucl. Phys. A510 (1990) 740.

[8] R. F. Frosch et al., Phys. Rev. 174 (1968) 1380.

[9] H. Kurasawa, H. Madokoro and T. Suzuki, to be published.

[10] T. Suda, private communication.
[1] D.Vautherin and D. M. Brink, Phys. Rev. C5 (1972) 626.

[2] B. D. Serot and J. D. Walecka, Adv. Nucl. Phys. 16 (1986) 1.

[3] W. Bertozzi, J. Friar, J. Heisenberg and J. W. Negele, Phys. Lett. 41B (1972) 408.

[4] L. D. Miller, Phys. Rev. C14 (1976) 706.

[5] C. J. Horowitz and B. D. Serot, Nucl. Phys. A368 (1981) 503. 